\title{
FLOCCULATION: A GENERAL AGGREGATION-FRAGMENTATION FRAMEWORK
}

\author{
Alex Sheremet ${ }^{1}$, Cihan Sahin ${ }^{2}$, Andrew J. Manning ${ }^{3,1}$ \\ We present a brief review of the key assumptions at the basis of the kinetic description of aggregation-fragmentation \\ processes. The goal is to provide a consistent foundation for using the aggregation-fragmentation equation as a frame- \\ work for modeling flocculation. The derivation presented here is illustrated using a simple form of the equation that \\ describes the dynamics of a 3-class distribution subject to elementary collisions of order 2, with arbitrary interaction \\ coefficients.
}

Keywords: flocculation; kinetic equations; aggregation-fragmentation

\section{INTRODUCTION}

The primary mineral component of cohesive muds are clay particles with a plate-like structure, and a diameter of less than $2 \mu \mathrm{m}$. Cohesion arises through a combination of electrostatic charging of primary particles and various biogenic polymer molecules which adhere to individual particles, and causes the aggregation of primary particles into larger agglomerates called flocs which behave as an identifiable sedimentary unit (e.g., Winterwerp and Van Kesteren, 2004; Mehta, 2013). Under typical estuarine conditions, most suspended particulate matter occurs in the form of flocs (Kranck and Milligan, 1992). In given environmental conditions, observations suggest that flocs have a wide distribution of sizes that can span over four orders of magnitude, from individual clay particles to stringer-type floc structures several $\mathrm{cm}$ in length. An individual floc might comprise up to of order $10^{6}$ primary particles. As a floc grows in size, its effective density (i.e., bulk density minus water density) generally decreases (e.g., Tambo and Watanabe, 1979; Klimpel and Hogg, 1986; Droppo et al., 2000). Because of the size dependency of the Stokes Law (Gregory, 1978; Mehta and Lott, 1987; Stolzenbach and Elimelich, 1994; Dyer and Manning , 1999; Winterwerp and Van Kesteren, 2004) the settling velocity of flocculated sediments is significantly greater than the constituent particles and can range over four orders of magnitude $(0.01 \mathrm{~mm} / \mathrm{s} \mathrm{up} \mathrm{to} \mathrm{cm} / \mathrm{s} ;$ Lick, 1994).

Flocculation, i.e., the formation of flocs, has long been recognized as a dynamic process (e.g., Winterwerp and Van Kesteren, 2004) driven by environmental conditions, primarily through flow-sediment interaction (e.g., Tsai et al., 1987; Burban, 1987; Burban et al., 1990; Manning, 2004). The physical and biochemical factors affecting flocculation include turbulence intensity, differential settling, sediment availability, primary-particle composition, salinity, and content of organic matter and polymers, and others (Burban et al., 1989; Lick et al., 1993; Manning and Dyer, 1999; Mietta et al., 2009; Manning et al., 2013; Parsons et al., 2016; Yang et al., 2016). Flocculation has a significant effect on deposition, erosion and consolidation rates, which makes it a primary mechanisms controlling sediment transport in fine-grained sedimentary environments. Because the characteristic floc size and settling velocity are considered key parameters for cohesive sediment transport models (Mehta and Lott, 1987), the interest in modeling the flocculation process has been growing.

All flocculation models are based on some version of the aggregation-fragmentation equation (AFE e.g., Drake, 1972; Ball and Carr, 1990), which expands the coagulation equation originally proposed by Smoluchovsky (1917). While the basic flocculation principles are generally agreed upon, the flocculation debate and contributions (e.g., McAnally and Mehta, 2000; Maggi et al., 2007; Lee et al., 2011; Verney et al., 2011) seem to be focused on the role and form of particular terms, such as turbulence-induced collision and breakup processes (Maggi et al., 2007), or shear aggregation (Verney et al., 2011). To reduce computational costs, some models focus on simplified distributions (e.g., bimodal, Lee et al., 2011). With a few exceptions (e.g., Lee et al., 2011), most models assume homogeneous and isotropic suspensions (so-called 0-D models), neglect settling effects and boundary sediment sources, and neglect all size-class interactions that involve more than 3 classes.

Remarkably, most authors caution about the effort to integrate the AFE in its basic form, and stress the need for numerically efficient simplifications (e.g., bimodal distribution of Lee et al., 2011). Simplifications, however, should preserve the essential behavior of the full AFE. Even the simple notion of "characteristic

\footnotetext{
${ }^{1}$ Engineering School for Sustainable Infrastructure and Environment, 365 Weil Hall, University of Florida, Gainesville FL 32611, USA

${ }^{2}$ Department of Civil Engineering, Yildiz Technical University, Esenler, Istanbul 34210, Turkey

${ }^{3}$ HR Wallingford Ltd, Coasts \& Estuaries Group, Wallingford, Oxfordshire, OX10 8BA, UK
} 
floc size" implies that "essential features" of the dynamics of the floc-size distribution can be represented by a low-dimensional model (a few size classes). Is this true? Under what conditions? What are these "essential features"? For example, one would expect low-dimensional models to work for equilibrium distributions. Do equilibrium distributions exist? What kind of equilibrium can be achieved (local or global)? While the effort to derive and validate coefficients for various AFE terms is important, working with truncations of the equation (e.g., 0-D models) has no solid justification beyond personal intuition, unless the general behavior of the system is understood. Comparisons with observations do not provide a compelling validation because: 1) field observations contain inherent forcing variability on multiple scales, and relaxation times associated with the floc-size distributions are not known; 2) it is almost impossible to control the forcing (most of the existing studies are based on laboratory experiments under controlled forcing conditions); and 3) current observations capabilities to resolve floc sizes are quite limited.

The AFE has been studied quite extensively in general (e.g., Drake, 1972; Ball and Carr, 1990; Connaughton and Krapivsky, 2010, and many others), but so far little is known about its behavior as a flocculation model. The goal of this paper is to derive the full AFE framework from the perspective of flocculation, and prove some basic properties, such as mass conservation and the existence of local-balance equilibrium for very simple cases. We hope that this will provide a general framework for the study of flocculation. While exploring the dynamics of the full AFE is a significant effort, we contend that it is an important step in understanding flocculation, defining the "essential features" of its dynamics, and providing a comprehensive validation framework for future numerically-efficient formulations.

\section{THE AGGREGATION-FRAGMENTATION FRAMEWORK}

Here we formalize the concepts of floc and flocculation, and set the foundation for the AFE framework. The model proposed below refers to the dynamics of aggregation/disaggregation of flocs and largely ignores primary particles. Instead of flocs, we will use below the term "particle".

\section{Particles}

A particle is a sedimentary unit that has long life time $T$ compared to the characteristic time $\tau$ of an aggregation/fragmentation process, i.e., $T \gg \tau$. The hypothesis of separation of time scales is fundamental to the kinetic description of the aggregation-fragmentation process. It implies that 1) particles have to be somewhat resilient to forces in the fluid, and 2) that primary particles should not be included in this definition. Indeed, assume that the concentration of primary particles is high enough that particles float in a "soup" of primary particles, and exchange continuously primary particles with the soup. If primary particles are allowed as components of the input/output state, the identity of a particle changes through absorbing/shedding of a single primary particle. Because the such exchange can be regarded as continuous, the lifetime of a particle shrinks to the same order of magnitude as the duration of collisions, violating the time-scale separation. If the characteristic mass of a primary particle is $\bar{m}$, the mass $m$ of a particle satisfies the relation $m \gg \bar{m}$. In other words, particles contain a large number, say $O\left(10^{5}\right)$, of primary particles (e.g., microflocs, $D<160 \mu \mathrm{m}$ are resistant to break-up by turbulent shear and are generally considered to be the building blocks of larger flocs; Eisma, 1986; Manning, 2001).

For numerical modeling purposes particles are divided into a discrete number of classes based on their mass. Denote by $\Delta m \gg \bar{m}$ the characteristic mass of smallest particles. The set of particles with mass

$$
\bar{m} \ll|m-k \Delta m| \leq \Delta m
$$

is called a $k$-class. We will approximate the mass of a $k$-particle as $m_{k}$. We will refer to particles from class $k$ as $k$-particles. If $p$ and $q$ are two classes such that $m_{p}<m_{q}, q$ and $p$ will be called upper, and lower class, respectively.

In some volume $V$ of fluid, let $M_{k}$ be the total mass of $k$-particles, and $N_{k}=M_{k} / m_{k}$ the number of $k$-particles in $V$. The total number of particles in $V$ is $N=\sum_{k} N_{k}$, and the total mass of sediment in $V$ is $M=\sum_{k} M_{k}=\sum_{k} N_{k} m_{k}$. The density of $k$-particles in $V$ can be defined in the standard way as

$$
\rho_{k}(\mathbf{r}, t)=\lim _{V_{\mathbf{r}} \rightarrow 0} \frac{M_{k}(t)}{V_{\mathbf{r}}}=n_{k}(\mathbf{r}, t) m_{k},
$$

where $V$ is a volume containing the point $\mathbf{r}=(x, y, z)$, and $n_{k}(\mathbf{r}, t)$ is the number of particles per unit volume. In Eq. 2 the limit is taken in a macroscopic sense (i.e., $V_{\mathbf{r}}$ contains a very large number of particles). In the 
absence of sources, the total mass $M$ of the sediment, and the total number of elementary particles in the system $M / m_{0}$ is are conserved. The total number of particles $N$ is not conserved.

\section{Collisions}

A collision is a type of aggregation/fragmentation event that occurs on a time scale $\tau$ and redistributes an input set of $N p_{i}$-particles, $i=1,2, \cdots, N$, into an output set of $M q_{j}$-particles $j=1, \cdots, M$, represented by the transformation

$$
P_{N}=\left\{p_{1}, p_{2}, \cdots, p_{N}\right\}=\left\{p_{i}\right\}_{N} \rightarrow Q_{M}=\left\{q_{1}, q_{2}, \cdots, q_{M}\right\}=\left\{q_{i}\right\}_{M} .
$$

This definition identifies types of collisions, and not individual, physical aggregation or fragmentation events. Individual physical collision events of the same type are indistinguishable. Unless specified otherwise, in the sequel, we shall denote classes and sets of classes by $p_{i}$ and $P_{N}$ for input, and $q_{i}$ and $Q_{M}$ for output. For simplicity, and where the meaning is clear, we will use the shorthand $P=\left\{p_{i}\right\}$, and $Q=\left\{q_{i}\right\}$, where the indices $i$ are understood to be dummy (local) indices that have their own, distinct range. The collision type is uniquely defined by the input $P_{N}$ and output $Q_{M}$ classes. Hence, a parameter $A$ associated with the collision type $P_{N} \rightarrow Q_{M}$ will be represented in the form $A_{P_{N}}^{Q_{M}}$, with the subscript denoting the input and the superscript denoting the output. Because input/output can consist of multiple particles from the same class, the classes $p_{i}$ and $q_{j}$ are not necessarily distinct. The order in the sets $P_{N}$ and $Q_{M}$ does not matter. Collisions conserve mass, and based on Eq. 1, also the sum of class indices:

$$
\sum_{j=1}^{M} m_{q_{j}}=\sum_{i=1}^{N} m_{p_{i}}, \quad \text { and } \quad \sum_{j=1}^{M} q_{j}=\sum_{i=1}^{n} p_{i}
$$

\section{THE AGGREGATION-FRAGMENTATION EQUATION}

The general aggregation-fragmentation equation describes the time evolution of the class distribution of sediment particles. From the Reynolds transport theorem, the rate of change of the total $k$-class mass $M_{k}(t)$ in volume $V(t)$ of boundary $S(t)$ is

$$
\frac{d M_{k}}{d t}=\int_{V} \frac{\partial \rho_{k}}{\partial t} d V+\oint_{S} \rho_{k} \mathbf{v}^{k} \cdot d \mathbf{A},
$$

where the density of $k$-class particles is given by Eq. $2, \mathbf{v}^{k}=\left(v_{1}^{k}, v_{2}^{k}, v_{3}^{k}\right)$ is the transport velocity of $k$-class particles assumed constant across the class, and $d \mathbf{A}$ is the vector element of area of $S$. The total derivative represents the mass change in class $k$ due to creation/destruction in the volume $V$ through collisions, while the surface integral represents the net flux of $k$-class mass through the boundary $S$. Dividing Eq. 5 by $m_{k}$ and switching to the differential formulation yields the equation for the rate of change of the number of $k$-particles in the unit volume

$$
\frac{d n_{k}}{d t}=\frac{\partial n_{k}}{\partial t}+\nabla \cdot\left(n_{k} \mathbf{v}^{k}\right)
$$

The rate of creation/destruction of $k$-particles can be expressed as the net flux across classes toward class $k$, i.e., formally as

$$
\frac{d n_{k}}{d t}=\mathcal{F}_{k},
$$

where $\mathcal{F}_{k}$ is the creation flux for class $k$ (negative is particles are destroyed), i.e., total number of $k$-particle created through collisions in the unit of time and unit of volume. Combining Eqs. 6 and 7 yields

$$
\frac{\partial n_{k}}{\partial t}+\nabla \cdot\left(n_{k} \mathbf{v}^{k}\right)=\mathcal{F}_{k}
$$

If $\mathcal{N}$ and $\mathcal{N}_{P_{N}}^{Q_{M}}$ are the total number of physical collision events and the total number of collisions events of type $P_{N} \rightarrow Q_{M}$ (Eq. 3) in the unit of volume per unit of time, then

$$
\mathcal{N}_{P_{N}}^{Q_{M}}=\mathcal{N} P_{P_{N}}^{Q_{M}}=\mathcal{N} W_{P_{N}}^{Q_{M}} \prod_{i=1}^{N} n_{p_{i}}
$$


where $\mathrm{P}_{P_{N}}^{Q_{M}}$ can be interpreted as the probability of a collision of type $P_{N} \rightarrow Q_{M}$, and we assumed that the number of physical collision events of this type is proportional to the number of particles $n_{p_{i}}$ in each of the input classes $p_{i}$. The normalized interaction coefficients $W_{P_{N}}^{Q_{M}}$ inherit the invariance to permutations of indices $\left(i_{1}, \cdots, i_{N}\right)$ and $\left(j_{1}, \cdots, j_{M}\right)$. Finally, the number of particles created can be calculated by examining the input and output of the collision. Let $\mu_{P, Q}^{k} \geq 0$ be the multiplicity of class $k$ in the input/output sets $P$ and $Q$, satisfying $\mu_{P}^{k}+\mu_{Q}^{k}>0$ (at least one is non-zero). Then, each physical collision event destroys $\mu_{P}^{k}$ and creates $\mu_{Q}^{k} k$-particles, therefore, the total flux of $k$-particles is

$$
\mathcal{F}_{k}=\sum_{N}\left(\mu_{Q}^{k}-\mu_{P}^{k}\right) \mathcal{N} W_{P_{N}}^{Q_{M}} \prod_{i=1}^{N} n_{p_{i}}
$$

Eq. 8 can be written as

$$
\frac{\partial n_{k}}{\partial t}+\nabla \cdot\left(n_{k} \mathbf{v}^{k}\right)=\mathcal{N} \sum_{N} \sum_{P, Q}\left(\mu_{Q}^{k}-\mu_{P}^{k}\right) W_{P_{N}}^{Q_{M}} \prod_{i=1}^{N} n_{p_{i}}
$$

where the summation on the right-hand side is taken over all possible $P \rightarrow Q$ collisions. Eq. 11 is a general discrete form of the AFE. It includes multiple-particle collisions with arbitrary input and output, as well as a basic advection mechanism for handling spatial inhomogeneities and transport (other mechanisms such as diffusion can be included in a straightforward way). The only restriction so far is the assumption of time-scale separation between particle life and collision scale. Without it, however, the concept of "particle" becomes difficult to define. Eq. 11 is seldom seen in this form, because of the difficulty to quantify the parameters that characterize arbitrary collisions. To become useful, the equation require further simplifications.

\section{Restriction to elementary, arbitrary-order collisions}

We will use the term elementary collision to denote a is a collision whose input or outcome (but not both) is a single particle, i.e., either $P_{N} \rightarrow Q_{1}$ or $P_{1} \rightarrow Q_{N}$ (compare with Eq. 3). The number $N$ of classes that either aggregate, or are the outcome fragments will be called the order of the collision. An elementary collision of order $N$ will be referred to as an $N$-collision.

A straightforward simplification of the general AFE (Eq. 11) is to assume that the probability of nonelementary collisions is much smaller that that of elementary collisions. Restricting to elementary collisions is equivalent assuming that aggregation and fragmentation can only occur separately. A direct consequence of this is that the fragments of the collision (either input or output) are in lower classes (smaller mass) than the whole. The total flux for class $k$ can be written explicitly in terms of aggregation and fragmentation events as

$$
\begin{aligned}
& \sum_{N} \sum_{P, Q}\left(\mu_{Q}^{k}-\mu_{P}^{k}\right) W_{P_{N}}^{Q_{M}} \prod_{i=1}^{N} n_{p_{i}}= \\
& \sum_{N}\left(\sum_{p, Q} \mu F_{p}^{Q_{N}} n_{p}+\sum_{P} A_{P_{N}}^{k} \prod_{i=1}^{N} n_{p_{i}}-\sum_{Q} F_{k}^{Q_{N}} n_{k}-\sum_{P, q} \mu A_{P_{N}}^{q}\left(n_{k}\right)^{\mu} \prod_{i=1}^{N-\mu} n_{p_{i}}\right)+\text { n.e.c. }
\end{aligned}
$$

where n.e.c. stands for the neglected non-elementary collisions terms. In Eq. 12 we dropped the sub/superscripts for the multiplicity $\mu$, and we replaced the generic notation $W$ for the interaction coefficient with $F$ (fragmentation) and $A$ (aggregation). Summation symbols indicate sums over all allowable classes and all allowable sets of input/output sets. For example, $\sum_{p, Q}$ sums over all values of class $p$ and all allowable sets of fragment classes $Q=\left\{q_{i}\right\}$. For single-e-class input/output, coefficients are indexed by the class rather than the set (e.g., $p$ instead of $P_{1}=\{p\}$ ). The meaning of the right-hand side terms is as follows. The first two terms represent creation fluxes for class $k$, resulting from two types of collisions:

1. $P_{N}=\left\{p_{1}, \cdots p_{N}\right\} \rightarrow Q_{1}=\{k\}$, with $p_{1}, \cdots p_{N}<k$ :

a set of $N$ particles from classes $p_{i}<k$ lower than class $k$, aggregate into a $k$-particle. The summation is carried over all possible collisions of this type, i.e., all number of fragments $N$, and all possible input sets $P_{N}$. 
2. $P_{1}=\{p\} \rightarrow Q_{N}=\left\{q_{1}, \cdots q_{N}\right\}$, with $q_{i_{1}}, \cdots, q_{i \mu}=k$, and $p, q_{i_{\mu+1}}, \cdots, q_{i_{N}} \neq k$ :

a $p$-particle $(p \neq k)$ breaks into $N$ particles, of which $\mu$ particles are of class $k\left(q_{i_{1}}, \cdots, q_{i \mu}=k,\right)$ and the rest $N-\mu$ are not in class $k\left(q_{i_{\mu+1}}, \cdots, q_{i_{N}} \neq k\right)$. The summation is carried over all the possible collisions of this type, i.e., all number of fragments $N$, all possible $p$-classes, and all possible output sets $Q_{N}$, The number $\mu \leq N$ is the multiplicity of class $k$ in the output set $Q_{N}$.

The last two terms represent destruction fluxes, also of two collision types

3. $P_{1}=\{k\} \rightarrow Q_{N}=\left\{q_{1}, \cdots q_{N}\right\}$, with, with $q_{1}, \cdots q_{N}<k$ :

a $k$-particle breaks into $N$ particles of classes $q_{j}<k$ lower than $k$. The summation is carried over all possible sets of output classes, i.e., all number of fragments $N$, and all possible output sets $Q_{N}$.

4. $P_{N}=\left\{p_{1}, \cdots p_{N}\right\} \rightarrow Q_{1}=\{q\}$, with $p_{i_{1}}, \cdots, p_{i \mu}=k$, and $q, p_{i_{\mu+1}}, \cdots, p_{i_{N}} \neq k$ :

a number $\mu$ of $k$-particles together with $N-\mu$ particles of classes $p_{i}$ aggregate into a $q$-particle. The summation is carried over all the possible collisions of this type, i.e., all number of fragments $N$, all possible input sets $P_{N}$. The number $\mu \leq N$ is the multiplicity of class $k$ in the input set $P_{N}$.

In the list above, cases 1 and 3 correspond to a $k$-particle being the "whole" that aggregates from, or fragments into sub-particles of lower classes. The cases 2 and 4 correspond to a $k$-particle being a fragment that either results from the fragmentation, or contributes to the aggregation into, some other particle of higher class. If the conservation of mass (Eq. 4) is taken into account, the fluxes can be written as

$$
\begin{aligned}
& \text { 1. } \sum_{P} A_{P_{N}}^{k} \prod_{i=1}^{N} n_{p_{i}}=\sum_{N \geq 2} \sum_{P_{N}} U_{P_{N}} A_{P_{N}}^{k}\left(\prod_{i=1}^{N} n_{p_{i}}\right) \delta\left(k-\sum_{i=1}^{N} p_{i}\right) \\
& \text { 2. } \sum_{p, Q} \mu F_{p}^{Q_{N}} n_{p}=\sum_{N \geq 2} \sum_{p} \sum_{Q_{N}} \mu_{k} F_{p}^{Q_{N}} n_{p} \delta\left(p-\sum_{i=1}^{N} q_{i}\right) \\
& \text { 3. } \sum_{Q} F_{k}^{Q_{N}} n_{k}=\sum_{N \geq 2} \sum_{Q_{N}} U_{Q_{N}} F_{k}^{Q_{N}} n_{k} \delta\left(k-\sum_{i=1}^{N} q_{i}\right) \\
& \text { 4. } \sum_{P, q} \mu_{P}^{k} A_{P_{N}}^{q} n_{k}^{\mu} \prod_{i=1}^{N-\mu} n_{p_{i}}=\sum_{N \geq 2} \sum_{q} \sum_{P_{N}} \mu_{k} A_{P_{N}}^{q}\left(\prod_{i=1}^{N} n_{p_{i}}\right) \delta\left(q-\sum_{i=1}^{N} p_{i}\right),
\end{aligned}
$$

where $\delta(a, b)=1$ if $a=b$ and 0 otherwise (Kronecker delta). In Eq. 13 the summation is broken down by the order of collision $\left(\sum_{N}\right)$, and by the input/output set (e.g., $\left.\sum_{P}\right)$. The multiplicity number $\mu_{k}$ represents the number of times class $k$ enters an input/output set. The number $U_{P_{N}}$ denotes the number of equivalent forms the input set $P_{N}$ appears in the sum $\sum_{P_{N}}$ and it depends on the way collisions are counted. For example, if the collisions are counted as in Eq. 13, by cycling through all the available classes, the number $U$ is the reciprocal of the number of permutations with repetitions (to allow for repeated indices) of a given input set, i.e.,

$$
\sum_{P_{N}} U_{P_{N}}=\sum_{p_{1}} \cdots \sum_{p_{N}} \frac{1}{N !} \prod_{i=1}^{N}\left(\mu_{p_{i}} !\right)
$$

where the number $\mu_{p_{i}}$ is the multiplicity of the value of index $p_{i}$. Collecting all the terms and dropping the formal distinction between input $(p)$ and output $(q)$ classes, Eq. 11 for elementary collisions becomes

$$
\begin{aligned}
& \frac{\partial n_{k}}{\partial t}+\nabla \cdot\left(n_{k} \mathbf{v}^{k}\right)=\mathcal{N} \sum_{N \geq 2}\left\{\sum_{P_{N}} U_{P_{N}}\left[A_{P_{N}}^{k}\left(\prod_{i=1}^{N} n_{p_{i}}\right)-F_{k}^{P_{N}} n_{k}\right] \delta\left(k-\sum_{i=1}^{N} p_{i}\right)\right. \\
& \left.-\sum_{q} \sum_{P_{N}} \mu_{k}\left[A_{P_{N}}^{q}\left(\prod_{i=1}^{N} n_{p_{i}}\right)-F_{q}^{P_{N}} n_{q}\right] \delta\left(q-\sum_{i=1}^{N} p_{i}\right)\right\}
\end{aligned}
$$

Eq. 15 is a version of the general AFE equation that takes into account elementary collisions of arbitrary order $N \geq 2$. 
EXAMPLE: AFE FOR ELEMENTARY COLLISIONS OF ORDER $N=2$

If the order of magnitude of the probability of $N$-collisions decreases as $N$ increases, for example, $\mathrm{P}_{\left\{p_{i}\right\}_{N}}^{k} \sim \mathrm{P}_{k}^{\left\{p_{i}\right\}_{N}}=O\left(\epsilon^{N}\right)$, where $\epsilon \ll 1$ a small parameter, then the lowest order collisions $(N=2)$ dominate the dynamics, and in the leading order Eq. 15 can be approximated by the 2-collision AFE equation

$$
\begin{aligned}
\frac{\partial n_{k}}{\partial t}+\nabla \cdot\left(n_{k} \mathbf{v}^{k}\right)= & \mathcal{N} \sum_{p, q} \frac{\left(\mu_{p} !\right)\left(\mu_{q} !\right)}{2}\left[A_{\{p, q\}}^{k} n_{p} n_{q}-F_{k}^{\{p, q\}} n_{k}\right] \delta(k-p-q) \\
& -\mathcal{N} \sum_{p, q} \mu_{k}\left[A_{\{k, p\}}^{q} n_{k} n_{p}-F_{q}^{\{k, p\}} n_{q}\right] \delta(k+p-q) .
\end{aligned}
$$

Eq. 16 is the preferred form of the AFE in the flocculation literature (e.g., Maggi et al., 2007; Verney et al., 2011).

\section{Equilibrium distributions}

The AFE (Eq. 11) is a kinetic equation similar to equations encountered in statistical physics (e.g., the celebrated Boltzmann equation Lifshitz and Pitaevskii, 1981), plasma physics (Kadomtsev, 1965), surface waves (Hasselmann, 1962), nonlinear optics and other fields of physics (Zakharov et al., 1992). Similar equations have been extensively studied (e.g., Nazarenko, 2011) and are integrated as a matter of routine, for example, in ocean wave forecasting (e.g., Cavaleri et al., 2007). The distributions described by these equations can achieve several types of stationarity (equilibrium) shapes. Two better known types are equilibrium states achieved through local balance (at class $k$, the creation flux exactly balances the destruction flux, i.e., $\mathcal{F}_{k}=0$ in Eq. 8), or through a constant flux across a range of classes (similar to the Kolmogorov inertial range in turbulence). The existence of global equilibrium is associated to power-law, self-similar class distributions similar to Kolmogorov-Zakharov spectra (Zakharov et al., 1992; Frisch, 1995; Nazarenko, 2011) but investigating them requires a more complicated analysis of the system of equations.

In contrast, the existence of local-balance-equilibrium distributions is simpler, as the condition required is to cancel the right-hand side of Eq. 11. We illustrate the principle for a simple possible AFE form, Eq. 16 with 3 classes, under the assumption of spatial homogeneity and isotropy, and constant environment conditions (i.e., constant interaction coefficients). Let the classes be $m_{k}=k m$, with $k=1,2,3$. Eq. 16 becomes

$$
\begin{aligned}
& \frac{1}{\mathcal{N}} \frac{d n_{1}}{d t}=-2 A_{\{1,1\}}^{2} n_{1}^{2}+2 F_{2}^{\{1,1\}} n_{2}-A_{\{1,2\}}^{3} n_{1} n_{2}+F_{3}^{\{1,2\}} n_{3} \\
& \frac{1}{\mathcal{N}} \frac{d n_{2}}{d t}=A_{\{1,1\}}^{2} n_{1}^{2}-F_{2}^{\{1,1\}} n_{1}-A_{\{2,1\}}^{3} n_{2} n_{1}+F_{3}^{\{2,1\}} n_{3}, \\
& \frac{1}{\mathcal{N}} \frac{d n_{3}}{d t}=A_{\{1,2\}}^{3} n_{1} n_{2}-F_{3}^{\{1,2\}} n_{3},
\end{aligned}
$$

In the way collisions are counted here, the right-hand side of the equation for class 3 appears twice in the equations, but with a factor

$$
U_{\{1,2\}}=\frac{\mu_{1} ! \mu_{2} !}{2 !}=\frac{1}{2}
$$

so it is counted only once. The conservation of the mass $M=\left(n_{1}+2 n_{2}+3 n_{3}\right) m$ of the system Eq. 17 is readily verified, since the all the right-hand side terms cancel in pairs

$$
\begin{gathered}
\frac{m_{1}}{\mathcal{N}} \frac{d}{d t}\left(n_{1}+2 n_{2}+3 n_{3}\right)=-2 A_{\{1,1\}}^{2} n_{1}^{2}+2 F_{2}^{\{1,1\}} n_{2}-A_{\{1,2\}}^{3} n_{1} n_{2}+F_{3}^{\{1,2\}} n_{3} \\
+2\left(A_{\{1,1\}}^{2} n_{1}^{2}-F_{2}^{\{1,1\}} n_{1}-A_{\{2,1\}}^{3} n_{2} n_{1}+F_{3}^{\{2,1\}} n_{3}\right)+3\left(A_{\{1,2\}}^{3} n_{1} n_{2}-F_{3}^{\{1,2\}} n_{3}\right)=0 .
\end{gathered}
$$

The local-balance equilibrium solution can also be found with ease. Setting all the class-creation/destruction fluxes to zero yields

$$
\begin{aligned}
& 0=-2 A_{\{1,1\}}^{2} n_{1}^{2}+2 F_{2}^{\{1,1\}} n_{2}-A_{\{1,2\}}^{3} n_{1} n_{2}+F_{3}^{\{1,2\}} n_{3} \\
& 0=A_{\{1,1\}}^{2} n_{1}^{2}-F_{2}^{\{1,1\}} n_{1}-A_{\{1,2\}}^{3} n_{2} n_{1}+F_{3}^{\{1,2\}} n_{3}, \\
& 0=A_{\{1,2\}}^{3} n_{1} n_{2}-F_{3}^{\{1,2\}} n_{3}
\end{aligned}
$$


From the last equation one obtains

$$
n_{3}=\frac{A_{\{1,2\}}^{3}}{F_{3}^{\{1,2\}}} n_{1} n_{2},
$$

and substituting into the first 2 equations yields

$$
n_{1}=n_{2}=\frac{F_{2}^{\{1,1\}}}{A_{\{1,1\}}^{2}}, \quad \text { hence } \quad n_{3}=\frac{A_{\{1,2\}}^{3}}{F_{3}^{\{1,2\}}}\left(\frac{F_{2}^{\{1,1\}}}{A_{\{1,1\}}^{2}}\right)^{2} \text {. }
$$

\section{SUMMARY}

In this paper we review briefly key assumptions in the derivation of the general AFE, and illustrated the points using a simple form of the equation that describes the dynamics of a 3-class distribution subject to elementary collisions of order 2 , with arbitrary interaction coefficients.

While the AFE has been used before as a model for flocculation, the significance of the form of the equation used, as well as the assumptions on which it is based are often if not misstated, at least overlooked, and often misunderstood. The AFE form for elementary collisions of order 2 (Eq. 16) is typically presented as the AFE (with no qualifications). As shown here, Eq. 16 is the fact a strongly simplified version of the general AFE (Eq. 11). While higher order versions of the AFE are no doubt more difficult to implement and use for practical applications, even in the case of Eq. 16, however, a good deal of ambiguity and misunderstanding is associated with the discussion of its scope and fundamental assumptions. The kinetic character (Eq. 1) of the AFE, whatever level of simplification, is often ignored. For example, Maggi et al. (2007) define a $k$-class as the type of particles that contain $k$ primary particles. The separation of time scales of physical interaction of particles and particle life-time imply that primary particles should not be considered as a class in themselves. McAnally and Mehta (2002) discuss at length the details of the physical interaction process and the circumstances that may lead to collisions of the type $P_{N} \rightarrow Q_{M}$ with $M>1$ and $M+N>1$. While the mechanism itself is likely important for defining the probability of such collisions, the AFE itself cannot include the description of the details of the interaction.

Although the family of kinetic equations to which the AFE belongs have been studied for a long time in other fields, very little is known about the properties of the AFE as a mathematical representation of flocculation processes. Equilibrium solutions have not been investigated even for its simplest form. We stress that this is not an indictment of previous research, which has mainly focused on the derivation of particular forms of the interaction coefficients corresponding to different aggregation and fragmentation mechanisms. Quite the contrary, we believe that previous work has laid a solid foundation for pursuing a better understanding of the complicated AFE dynamics.

\section{ACKNOWLEDGMENTS}

We wish to thank Professor A.J. Mehta for the encouragement and helpful discussions on the topic of flocculation and the AFE framework. Professor Manning's contribution to this paper was partly funded by the HR Wallingford Company Research project 'FineScale - Dynamics of Fine-grained Cohesive Sediments at Varying Spatial and Temporal Scales' (DDY0523).

\section{References}

Ball, J.M., and J. Carr. 1990. The Discrete Coagulation-Fragmentation Equations: Existence, Uniqueness, and Density Conservation, Journal of Statistical Physics, 61(1/2), 203-234.

Burban, P.Y. 1987. The flocculation of fine-grained sediments in estuarine waters, MSc. thesis, Dep. of Mech. Eng. Univ. of Calif., Santa Barbara, USA.

Burban, P.-Y., W. Lick, and J. Lick. 1989. The flocculation of finegrained sediments in estuarine waters, Journal of Geophysical Research, 94, 8323-8330.

Burban, P.-Y., Y.-J. Xu, J. McNeil, and W. Lick. 1990. Settling speeds of flocs in freshwater and seawater, Journal of Geophysical Research, 95, 18,213- 18,220. 
Cavaleri, L., J.-H.G.M. Alves, F. Ardhuin, A. Babanin, M. Banner, K. Belibassakis, M. Benoit, M. Donelan, J. Groeneweg, T.H.C. Herbers, P. Hwang, P.A.E.M. Janssen, T.Janssen, I.V. Lavrenov, R. Magne, J. Monbaliu, M. Onorato, V. Polnikov, D. Resio, W.E. Rogers, A. Sheremet, J. McKee Smith, H.L. Tolman, G. van Vledder, J. Wolf, I. Young. 2007. Wave modelling - The state of the art, Progress in Oceanography, $75,603-674$.

Connaughton, C. and P.L. Krapivsky. 2010. Aggregation-fragmentation processes and decaying three-wave turbulence, Physical Review E, 81, 035303(R) DOI: 10.1103/PhysRevE.81.035303

Drake, R. 1972. In Topics in Current Aerosol research, G.M. Hidy and J.R. Brock, eds. (Pergamon Press, Oxford).

Droppo, I.G., D. Walling, and E. Ongley. 2000. The influence of floc size, density and porosity on sediment and contaminant transport. Journal of the National Centre for Scientific Research, 4, 141-147.

Dyer, K. R., A.J. Manning. 1999. Observation of size, settling velocity and effective density of flocs, and their fractal dimensions, Journal of Sea Research, 41, 87-95.

Eisma, D. 1986. Flocculation and de-flocculation of suspended matter in estuaries, Netherlands Journal of Sea Research, 20 (2/3), 183-199.

Frisch, U. 1995. Turbulence: The Legacy of A. N. Kolmogorov. Cambridge University Press. ISBN 9780521457132 .

Gregory, J. 1978. Effects of polymers on colloid stability. In: The Scientific Basis of Flocculation (Ed. Ives, K.J.), Pub. Sijthoff \& Noordhoff, Alphen aan den Rijn, The Netherlands: 89-99.

Hasselmann, K. 1962. On the nonlinear energy transfer in a gravity wave spectrum, Journal of Fluid Mechanics, 12, 481-500.

Kadomtsev, B.B. 1965. Plasma Turbulence, Academic Press, New York.

Klimpel R.C. and R. Hogg. 1986. Effects of flocculation conditions on agglomerate structure, Journal of Colloid Interface Science, 113, 121-131.

Kranck, K. and T.G. Milligan. 1992. Characteristics of suspended particles at an 11-hour anchor station in San Francisco Bay, California, Journal of Geophysical Research, 97, 11373-11382.

Lee, B.J., E. Toorman, F.J. Molz, and J.Wang J. 2011. A two-class population balance equation yielding bimodal flocculation of marine or estuarine sediments, Water Research, 45, 2131-2145.

Lick, W. 1994. Modelling the transport of sediment and hydrophobic contaminants in surface waters, $U$. S. / Israel Workshop on monitoring and modelling water quality, May 8-13, 1994, Haifa, Israel.

Lick, W., H. Huang, and R. Jepsen. 1993. Flocculation of fine-grained sediments due to differential settling, Journal of Geophysical Research, 98 (C6): 10,279-10,288.

Lifshitz, E.M. , and L.P. Pitaevskii. 1981. Physical Kinetics, Pergamon Press, Oxford, 1981.

Maggi, F., F. Mietta, and J.C. Winterwerp. 2007. Effect of variable fractal dimension on the floc size distribution of suspended cohesive sediment, Journal of Hydrology, 343(1-2), 43-55

Manning, A.J. and K.R. Dyer. 1999. A laboratory examination of floc characteristics with regard to turbulent shearing, Marine Geology, 160: 147-170.

Manning, A.J. 2001. A study of the effects of turbulence on the properties of flocculated mud, Ph.D. Thesis. Institute of Marine Studies, University of Plymouth, 282p.

Manning, A.J. 2004. The observed effects of turbulence on estuarine flocculation. In: P. Ciavola and M. B. Collins (Eds), Sediment Transport in European Estuaries, Journal of Coastal Research, SI 41, 90-104. 
Manning, A.J., J.R. Spearman, R.J.S. Whitehouse, E.L. Pidduck, J.V. Baugh, and K.L. Spencer. 2013. Laboratory assessments of the flocculation dynamics of mixed mud: sand suspensions. In: Manning, A.J. (Ed.), Sediment Transport Processes and their Modelling Applications, Intech, Rijeka, Croatia. ISBN: 978-953-51-1039-2, pp. 119-164. http://dx.doi.org/10.5772/3401.

McAnally, W. H., and A.J. Mehta. 2000. Aggregation rate of fine sediment, Journal of Hydraulic Engineering, 126, 883-892.

McAnally, W. H., and A.J. Mehta. 2002. Significance of aggregation of fine sediment particles in their deposition, Estuarine, Coastal and Shelf Science, 54: 643-653.

Mehta, A.J. and J.W. Lott. 1987. Sorting of fine sediment during deposition, Proc. Specialty Conf. Advances in Understanding Coastal Sediment Processes, Am. Soc. Civ. Eng., New York, pp. 348-362.

Mehta, A.J. 2013. An Introduction to Hydraulics of Fine Sediment Transport, Advanced Series on Ocean Engineering: Volume 38, Springer, 1060pp.

Mietta F., C. Chassagne and J.C. Winterwerp. 2009. Shear-induced flocculation of a suspension of kaolinite as function of $\mathrm{pH}$ and salt concentration, Journal of Colloid and Interface Science, 336:134-141.

Nazarenko, S. 2011. Wave Turbulence, Lecture Notes in Physics, Vol. 825, Springer, 279 pp.

Parsons, D.R., R.J. Schindler, J.A. Hope, J. Malarkey, J.H. Baas, J. Peakall, A.J. Manning, L. Ye, S. Simmons, D.M. Paterson, R.J. Aspden, S.J. Bass, A.G. Davies, I.D. Lichtman, and P.D. Thorne. 2016. The role of biophysical cohesion on subaqueous bed form size. Geophysical Research Letters, 43:1566-1573. http://dx.doi.org/10.1002/ 2016GL067667.

Smoluchowski, M. 1917.Versuch einer mathematischen theorie des koagulations- kinetik kolloid losungen, Zeitschrift fur Physikalische Chemie, 92,129-168.

Stolzenbach, K.D. and M. Elimelich. 1994. The effect of density on collisions between sinking particles: implications for particle aggregation in the ocean, Journal of Deep Sea Research I, 41 (3):469-483.

Tambo, N. and Y. Watanabe. 1979. Physical characteristics of flocs-I. The floc density function and aluminium floc, Water Research, 13, 409-419.

Tsai, C.H., S. Iacobellis, and W. Lick. 1987. Flocculation of fine-grained lake sediments due to a uniform shear stress, Journal of Great Lakes Research, 13: 135-146. doi:10.1016/S0380- 1330(87)71637-2.

Verney, R., R. Lafite, and J. Brun-Cottan. 2011. Behaviour of a floc population during a tidal cycle: laboratory experiments and numerical modelling. Continental Shelf Research, 31, S64-S83.

Winterwerp, J.C. and W.G.M. van Kesteren. 2004. Introduction to the physics of cohesive sediment in the marine environment. Developments in Sedimentology, 56, van Loon, T. (Ed.), Amsterdam: Elsevier, $466 \mathrm{p}$.

Yang R., H. Li, M. Huang, H. Yang, A. Li. 2016. A review on chitosan-based flocculants and their applications in water treatment, Water Research, 15 (95), 59-89.

Zakharov, V.E., V.S. L'vov, G. Falkovich. 1992. Kolmogorov Spectra of Turbulence, Springer, Berlin. 